\title{
Self Through the Other
}

Production, Circulation and Reception in Italy

of Sixteenth-Century Printed Sources on Japan

Sonia Favi

\section{Appendix Sixteenth-Century Vernacular Italian Letter-Books about Japan: a Bibliography}

In Italy, the following books including letters by Jesuit missionaries stationed in Japan were published between 1552 and 1601. These include both first editions, and reprinted editions of earlier works. Some books consist in but one letter, but most of them are collections. As a whole, the letter-books include a total of about a hundred letters.

All texts listed below are, as already mentioned, in the octavo book-format.

Avisi particolari delle Indie di Portogallo. Ricevuti in questi doi anni del 1551. \& 1552. da li reverendi padri de la Compagnia de Iesu, dove fra molte cose mirabili, si vede delli paesi delle genti, \& costumi loro \& la grande conversioue di molti populi, che cominciano a ricevere il lume della santa fede \& relligione christiana.

Rome: Valerio Dorico, et Luigi fratelli Bressani, 1552

Consistencies found in Italian libraries: 11

Nuoui auisi delle Indie di Portugallo riceuuti questo anno del 1553. doue si tratta della conuersione di molte persone principali \& tra li altri d'un re signore de 11000. isole, con vna descrittione delli costumi de i giaponesi nostri antipodi \& come loro riceuono la nostra santa fede.

Rome: Valerio Dorico, et Luigi fratelli Bressani, 1553

Consistencies found in Italian libraries: 2

Avisi particolari delle Indie di Portogallo. Nouamente hauuti questo anno del 1555 da li R. padri della Compagnia di Iesu doue si ha informatione delle gran cose che si fanno per augmento de la santa fede. Con la descriptione e costumi del Regno de la China, \& altri paesi incogniti nouamente trouati.

Rome: Antonium Bladum, 1556

Consistencies found in Italian libraries: 5 
Diversi avisi particolari dall'Indie di Portogallo ricevuti, dall'anno 1551. sino al 1558. dalli Reverendi padri della compagnia di Giesv. Dove s'intende delli paesi, delle genti, et costumi loro, et la grande conversione di molti popoli, che hanno ricevuto il lume della santa fede, et religione Christiana. Tradotti nuouamente dalla lingua Spagnuola nella Italiana.

Venice: Michele Tramezzino, [1558 $]^{1}$

Consistencies found in Italian libraries: 1

Venice: Michele Tramezzino, 1565

Consistencies found in Italian libraries: 1

Nuovi avisi dell'Indie di Portogallo, ricevuti dalli Reverendi Padri della compagnia di Giesu, tradotti dalla lingua Spagnuola nell'Italiana.

Venice: Michele Tramezzino, 1559.

Consistencies found in Italian libraries: 22

Venice: Tramezzino, 1568

Consistencies found in Italian libraries: 1

Nuovi avisi dell'Indie di Portogallo, Riceuuti dalli Reuerendi Padri della compagnia di Giesu, tradotti dalla lingua Spagnuola nell'Italiana, Terza parte. Col priuilegio del Sommo Pontefice, et dell'Illustrissimo Senato Veneto per anni XX.

Venice: Michele Tramezzino, 1562

Consistencies found in Italian libraries: 1

Nuovi avisi dell'Indie di Portogallo, Venuti nuouamente dalli R. Padri della compagnia di GIESV, \& tradotti dalla lingua Spagnuola nella Italiana. Quarta parte. Col priuilegio del Sommo Pont. Pio IIII. Et dell'Illustriss. Senato Veneto per anni XX.

Venice: Tramezzino, 1580

Consistencies found in Italian libraries: 1

Lettere del Giapone de gli anni 74, 75, \& 76. Scritte dalli reuerendi padri della Compagnia di Giesu, \& di portughese tradotte nel volgare italiano.

Rome: Zanetti, 1578

Consistencies found in Italian libraries: 5

1 The publication date is tentative, derived from the preface. 
Rome: Zanetti, 1579

(included in some of the bibliographies, but no actual consistencies found)

Naples: eredi di Matteo Cancer, 1580

Consistencies found in Italian libraries: 1

Lettere del Giappone scritte dalli reuerendi Padri della Compagnia di Giesu. Dell'anno 1577.

Brescia: Giacomo e Policreto Turlini, 1580

Consistencies found in Italian libraries: 8

Naples: eredi di Matteo Cancer, 1580 (with the alternative title Lettere del Giappone dell' anno MDLXXVII scritte dalli reuerendi padri della Compagnia di Giesù)

Consistencies found in Italian libraries: 1

Lettere dell'India orientale, Scritte da' Reuerendi Padri della Compagnia di Giesv'. Nelle queli si scopre la grande arte vsata de gli istessi, per liberar l'anime degli infideli Indiani dalla potestà del nimico infernale, et ridurle alla nostra santa fede. Nouamente stampate, \& ampliate in molti luoghi, $\&$ ricorrette con diligenza.

Venice: Antonio Ferrari, 1580

Consistencies found in Italian libraries: 11

Alcvne lettere delle cose del Giappone. Dell'anno 1579. Insino al 1581.

Rome: Zanetti, 1584

Consistencies found in Italian libraries: 15

Rome: Zanetti, 1584 (with the alternative title Alcune lettere delle cose del Giappone. Scritte da' reuerendi Padri della Compagnia di Iesu. Dell'anno 1579. insino al 1581)

Rome: Zanetti, 1584

Consistencies found in Italian libraries: 20

Naples: Orazio Salviani e Cesare Cesari, 1584 (with the alternative title Alcune lettere delle cose del Giappone. Scritte da' reuerendi padri della Compagnia di Iesu. Dell'anno 1579 infino al 1581)

Consistencies found in Italian libraries:1 
Brescia: Vincenzo Sabbio, 1584 (with the alternative title Alcune lettere delle cose del Giappone, paese del mondo nouo, dell'anno 1579 insino al 1581)

Consistencies found in Italian libraries: 6

Milan: Pacifico Ponte, 1584

Consistencies found in Italian libraries: 7

Venice: Giolito, 1585

Consistencies found in Italian libraries: 5

Lettera annale portata di novo dal Giapone delle cose ivi successe l'anno M D LXXXII.

Venice: Giolito, 1585

Consistencies found in Italian libraries: 11

Milan: Pacifico Ponte, 1585 (with the alternative title Lettera annale delle cose del Giappone del 1582)

Consistencies found in Italian libraries: 11

Rome: Zanetti, 1585 (with the alternative title Lettera annale delle cose del Giappone del 1582)

Consistencies found in Italian libraries: 17

Avisi del Giapone de gli anni MDLXXXII, LXXXIII et LXXXIV. Con alcuni altri della Cina dell'LXXXIII e LXXXIV. Cauati dalle lettere della Compagnia di Giesù .

Rome: Zanetti,1586

Consistencies found in Italian libraries: 13

Rome: Zanetti, 1586

Consistencies found in Italian libraries: 29

Milan: Pacifico Ponte, 1586

Consistencies found in Italian libraries: 5

Venice: Giolito, 1586 (with the alternative title Nvovi avvisi del Giapone con alcvni altri della Cina, del LXXXIII, et LXXXIV cavati dalle lettere della Compagnia di Giesv')

Consistencies found in Italian libraries: 1 
Venice: Giolito, 1586 (with the alternative title Nvovi avvisi del Giapone con alcvni altri della Cina, del LXXXIII, et LXXXIV cavati dalle lettere della Compagnia di Giesv')

Consistencies found in Italian libraries: 1

Viaggio nell'India Orientale.

Venice: Andrea Muschio, 1587 (included in some of the bibliographies, but no actual consistencies found)

Avvisi della Cina et Giapone del fine dell'anno 1586. Con l'arriuo delli signori Giaponesi nell'India. Cauati dalle lettere della Compagnia di Giesù. Riceuute il mese d'ottobre 1588.

Rome: Zanetti, 1588

Consistencies found in Italian libraries: 12

Rome: Zanetti, 1588 (with the alternative title Auuisi della Cina et Giapone del fine dell'anno 1587. Con l'arriuo delli signori giaponesi nell'India. Cauati dalle lettere della Compagnia di Giesù. Riceuute il mese d'ottobre 1588)

Consistencies found in Italian libraries: 5

Rome: Zanetti, 1588

(included in some of the bibliographies, but no actual consistencies found)

In Roma e poi Verona: Discepolo Girolamo, 1588

Consistencies found in Italian libraries: 3

Milan: Pacifico Ponte, 1588

Consistencies found in Italian libraries: 2

Naples: Horatio Salviani, 1588

Consistencies found in Italian libraries: 2

Venice: Giolito, 1588 (with the alternative title Auuisi della Cina, et Giapone del fine dell'anno 1587. Con l'arriuo de' signori giaponesi nell'India. Cauati dalle lettere della Compagnia di Giesù, riceuute il mese d'ottobre 1588)

Consistencies found in Italian libraries: 7

Anversa: Christoforo Plantino, 1588 (included in some of the bibliographies, but no actual consistencies found) 
Brescia: Vincenzo Sabbio, 1588 (included in some of the bibliographies, but no actual consistencies found)

Milan: Pacifico Ponte, 1589

Consistencies found in Italian libraries: 3

Raguaglio d'vn notabilissimo naufragio, cauato d'vna lettera del padre Pietro Martinez, scritta da Goa al molto reuerendo P. generale della Compagnia di Giesu alli 9. di Decembre 1586

Rome: Zanetti, 1588

Consistencies found in Italian libraries: 8

Venice: Giolito, 1588

Consistencies found in Italian libraries: 7

Milan: Pacifico Ponte, 1588

Consistencies found in Italian libraries: 3

Raccolta di molti auuisi del Giapone dell'anno 1582 fin all'87. Doue si tratta del progresso della fede christiana, delle varie riuolutioni, e mutationi de' Stati di quel paese, d'vna gran persecutione contra i fedeli, \& altre cose notabili. Con alcun'altri auuisi della China dell'anni 83. \& 84. Cauati dalle lettere della Compagnia di Giesu

Rome: Zanetti, 1590.

Consistencies found in Italian libraries: 1

Lettera annale del Giapone scritta al padre della Gompagnia [sic!] di Giesv alli XX. di Febraio M.D.LXXXVIII.

Rome: Zanetti, 1590

Consistencies found in Italian libraries: 20

Palermo: Giovanni Antonio De Franceschi, 1590

Consistencies found in Italian libraries: 1

Brescia: Vincenzo Sabbio, 1590 (with the alternative title Lettera annale del Giapone scritta, al padre generale della Compagnia di Giesù alli 20 di febraio 1588. Con l'auiso ancora dell'arriuo delli signori giaponesi, all'isola di Macao, del regno della China)

Consistencies found in Italian libraries: 5 
Milan: Pacifico Ponte, 1590 (with the alternative title Lettera annale del Giapone scritta al padre generale della Compagnia di Giesù Alli 20. di febraro 1588)

Consistencies found in Italian libraries: 6

Lettere del Giapone, et della Cina de gl'anni M. D. LXXXIX \& M. D. XC. Scritte al r.p. generale della Compagnia di Giesu.

Rome: Zanetti, 1591

Consistencies found in Italian libraries: 18

Milan: Pacifico Ponte, 1592

Consistencies found in Italian libraries: 3

Venice: Giovanni Battista Ciotti, 1592

Consistencies found in Italian libraries: 8

Brescia: Vincenzo Sabbio, 1592

Consistencies found in Italian libraries: 4

Ragguaglio d'alcune missioni delle Indie Orientali, \& Occidentali. Cavato da alcun auuisi scritti gli anni 1590. et 1591.

Rome: Zanetti, 1592

Consistencies found in Italian libraries: 10

Rome: Zanetti, 1592

Consistencies found in Italian libraries: 7

Rome, Turin: Zanetti, 1593

Consistencies found in Italian libraries: 3

Napoli: Giovanni Giacomo Carlino e Antonio Pace, 1593

Consistencies found in Italian libraries: 1

Bologna: Benacci, 1593 (with the alternative title Ragguaglio d'alcuni auisi notabili dell'Indie Orientali, \& Occidentali. Con l'arriuo delli signori ambasciatori Giaponesi alli loro stati. Cauato da alcune lettere scritte gli anni 1590. \& 1591. Da i PP. Pietro Martinez prouinciale dell'India Orientale, Giouanni d'Atienza prouinciale del Peru, Pietro Diaz prouinciale del Messico)

Consistencies found in Italian libraries: 2 
Copia di due lettere annue scritte dal Giapone del 1589. \& 1590. L'vna dal p. viceprouinciale al $p$. Alessandro Valignano, l'altra dal p. Luigi Frois al p. Generale della Compagnia di Giesu. Et dalla spagnuola nella italiana lingua tradotte dal p. Gasparo Spitilli della Compagnia medesima

Rome: Zanetti, 1593.

Consistencies found in Italian libraries: 12

Milan: Pacifico Ponte, 1593

Consistencies found in Italian libraries: 3

Brescia: Policreto Turlino, 1593

Consistencies found in Italian libraries: 1

Brescia: Policreto Turlino, 1598

Consistencies found in Italian libraries: 1

Lettera del Giapone degli anni 1591. Et 1592. Scritta al R.P. generale della Compagnia di Giesu.

Rome: Zanetti, 1595

Consistencies found in Italian libraries: 11

Milan: Pacifico Ponte, 1595

Consistencies found in Italian libraries: 2

Venice: Giovanni Battista Ciotti, 1595

Consistencies found in Italian libraries: 4

Mantova: Francesco Osanna, 1595

Consistencies found in Italian libraries: 1

Lettera annua del Giapone del marzo del 1593, sino al marzo del 94.

Rome: Zanetti, 1597

Consistencies found in Italian libraries: 10

Milan: Pacifico Ponte, 1597

Consistencies found in Italian libraries: 1

Copia di due lettere scritte dal P. Organtino bresciano della Compagnia di Giesu dal Meaco del Giapone. Al molto r. in Christo p.n. il p. Claudio Acquauiua preposito generale. Tradotte dal p. Gio. Battista Peruschi romano della medesima Compagnia. 
Rome: Zanetti, 1597

Consistencies found in Italian libraries: 12

Milan: Pacifico Ponte, 1597

Consistencies found in Italian libraries: 5

Verona: Girolamo Discepolo, 1597

Consistencies found in Italian libraries: 1

Ragguaglio della morte di Quabacondono, scritta dal p. Luigi Frois della Compagnia di Giesu, dal Giappone nel mese d'ottobre del 1595. Et dalla portoghesa nella lingua italiana tradotta dal P. Gasparo Spitilli di Campli.

Rome: Zanetti, 1598

Consistencies found in Italian libraries: 11

Copia d'vna lettera annua scritta dal Giapone nel 1595. al r. p. Claudio Acquauiua generale della Compagnia di Giesu. Et dalla portoghesa nella lingua italiana tradotta dal p. Gasparo Spitilli.

Rome: Zanetti, 1598

Consistencies found in Italian libraries: 11

Milan: Pacifico Ponte, 1598

Consistencies found in Italian libraries: 5

Relazione mandata da don Francesco Teglio gouernatore, e capitano generale dell'isole Filippine, intorno al martirio de i sei frati spagnuoli, dell'Ordine di San Francesco dell'osseruanza. Crocifissi nel Giappone l'anno 1597, con venti altre persone giapponese che con esso loro morirono, animati e conuertiti da gl'istessi santi frati, nella loro predicazione. In lingua spagnuola castigliana stampata in Siuiglia, e nell'italiana fauella tradotta dal r.p. frat'Angelo Celestino theologo, e predicatore nel Duomo di Firenze.

Rome: Niccolò Muzi, 1598

Consistencies found in Italian libraries: 1

Venice: Marcello Iseppo, 1598

Consistencies found in Italian libraries: 1

Urbino: Bartolomeo e Simone Ragusi, 1598

Consistencies found in Italian libraries: 1 
Roma: Francesco Osanna, 1598

Consistencies found in Italian libraries: 1

Napoli: Giacomo Carlino, 1599

Consistencies found in Italian libraries: 1

Relatione della gloriosa morte di ventisei posti in croce per comandamento del re di Giappone, alli 5. di febraio 1597. de' quali sei furono religiosi di S. Francesco, tre della Compagnia di Giesu, \& dicisette christiani Giapponesi, mandata dal p. Luigi Frois alli 15. di marzo al r. p. Claudio Aquauiua e fatta in italiano dal p. Gasparo Spitilli di Campli.

Rome: Zanetti, 1599

Consistencies found in Italian libraries: 6

Rome: Zanetti, 1599

Consistencies found in Italian libraries: 4

Bologna: eredi di Giovanni Rossi, ad instanza di Gasparo Bindoni, 1599 Consistencies found in Italian libraries: 4

Milan: Pacifico Ponte, 1599

Consistencies found in Italian libraries: 3

Trattato d'alcuni prodigii occorsi l'anno 1596. nel Giappone. Mandato dal p. Luigi Frois, della Compagnia di Giesu.

Rome: Zanetti, 1599

Consistencies found in Italian libraries: 3

Milano: Pacifico Ponte

Consistencies found in Italian libraries: 4

Lettera annua del Giappone dell'anno 1596. Scritta dal p. Luigi Froes, al r.p. Claudio Acquauiua generale della Compagnia di Giesù. Tradotta in italiano dal p. Francesco Mercati romano della stessa Copmpagnia.

Rome: Zanetti, 1599

Consistencies found in Italian libraries: 11

Milan: Pacifico Ponte, 1599

Consistencies found in Italian libraries: 2 
Venice (Padua): Francesco Bolzetta, 1599

Consistencies found in Italian libraries: 10

Relatione del martirio, che sei padri scalzi di San Francesco et venti Giaponesi christiani patirono nel Giapone l'anno 1597. Scritta dal R.P. fra Gio. di Santa Maria \& tradotta dalla lingua spagnuola nella italiana, per ordine del R.P. fra Gioseppe di Santa Maria.

Roma: Niccolò Muzi, 1599

Consistencies found in Italian libraries: 2

Napoli: Antonio Pace, 1600

Consistencies found in Italian libraries: 1 
